\title{
Genetik Algoritma ile İlerleyen Tür Tip 2 Sansürlü Örneklemlerde Weibull Dağılımının Parametrelerinin En Çok Olabilirlik Tahmini
}

\author{
${ }^{*}$ Aydin Karakoca, ${ }^{2}$ Ahmet Pekgör \\ Necmettin Erbakan Üniversitesi Fen Fakültesi İstatistik Bölümü Konya, Türkiye \\ 1akarakoca@konya.edu.tr, \\ 2apekgor@konya.edu.tr, \\ Research Paper \\ Arrival Date: 10.08 .2018 \\ Accepted Date: 27.11.2018
}

$\ddot{O} z$

Bu çalışmada Weibull dağılımına sahip ilerleyen tür tip 2 sansürlü örneklemlerde parametre tahmini probleminde Newton yöntemine alternatif bir çözüm önerilmiştir. Newton yöntemi en çok olabilirlik tahmininde sıklıkla kullanılmaktadır. Newton yöntemi popüler olmasına rağmen en büyük dezavantajı en az iki kez türevlenebilir fonksiyonlar için kullanılabilmesidir. Olabilirlik fonksiyonu sansürlü örneklemlerde tam örneklemlere göre fonksiyonel olarak daha kompleks bir yapıda olduğundan, türev ve diğer hesaplamalar nispeten daha karışıktır. Bu çalışmada en çok olabilirlik yönteminde elde edilen denklem sisteminin çözümü için Newton metodunun kullanımındaki kısıtlamalara bir alternatif olarak Genetik Algoritma önerilmiştir. Detaylı bir simülasyon çalışması yardımıyla yan ve hata kareler ortalaması ile iki yöntemin performansları değerlendirilmiştir. Simülasyon sonuçlarına göre önerilen yöntemin karşılaştırılan tüm durumlar için ölçek parametresi için daha iyi sonuçlar verdiği, şekil parametresi için ise yanlar açısından sonuçların benzer olduğu ancak hata kareler ortalamasına göre bazı sansür şemaları için Newton yönteminin iyi sonuç verdiği bulunmuştur.

Anahtar Kelimeler: İlerleyen Tür Tip 2 Sansürleme, Weibull Dağılımı, Genetik Algoritma

\section{Maximum Likelihood Estimation of the Parameters of Progressively Type-2 Censored Samples From Weibull Distribution Using Genetic Algorithm}

\author{
${ }^{* 1}$ Aydın Karakoca, ${ }^{2}$ Ahmet Pekgör \\ Department of Statistics, Faculty of Science, Necmettin Erbakan University, Konya Turkey \\ ${ }^{1}$ akarakoca@konya.edu.tr \\ 2apekgor@konya.edu.tr
}

\begin{abstract}
In this study we suggested an alternative solution to the parameter estimation problem of the Weibull distribution based on progressively Type-II censored samples with Newton method. Newton is one of the widely used methods for solving the system of equations especially in maximum likelihood estimation. Even though it is popular, the biggest disadvantage of the Newton method is that it is a valid method for only functions that derivativable at least two times. Since the likelihood functions are in more complex form for censored samples than in full samples, calculations of derivatives and related processes are more complicated. We proposed to use the Genetic Algorithm an alternative to the limitations of the Newton method in solution of system of equations in maximum likelihood estimation. Performance of these methods are evaluated by the simulated bias and mean square error criteria by an intensive simulation study. Simulation results of the study showed that the suggested method give better results than Newton method for scale parameter for all conditions. Also shape parameter results for simulated biases are similar for GA and Newton method but Newton has better mean squared error values for some censoring schemes.
\end{abstract}

Keywords: Progressive Type-2 Censoring, Weibull Distribution, Genetic Algorithm

Corresponding Author :*1 Department of Statistics, Faculty of Science, Necmettin Erbakan University, Konya, Turkey,

akarakoca@konya.edu.tr 


\section{INTRODUCTION}

It is a common situation that not to obtain all the observations completely in many studies such as statistics, engineering, economics and medical researches. Data obtained such studies are called censored samples. Such for example, in a medical study, some observations may not be obtained completely from various reasons such as the death of some patients or dropped out from the treatment. In many lifetesting studies, exact failure times of units may not be obtained completely by the experimenter in terms of time or cost constraints, similar examples can be expanded anyway.

Type-I and Type-II are widely used two popular censoring schemes in practice, especially life testing experiments. During the observation of $\mathrm{n}$ items in an experiment, Type-I and Type II censoring are defined as by measuring failure times and number of items, respectively. In Type-I and TypeII censoring scheme, the experiment ends either at prespecified time $T$ or number of failures $m(m \leq n)$ achieved.

Progressive censoring gives more flexibility to researchers compared to conventional Type-I and Type-II censoring. Consider testing $\mathrm{n}$ units on a life-test with censoring scheme $\underline{r}=\left(r_{1}, r_{2}, \cdots, r_{m}\right) . r_{i},(i=1,2 \cdots, m)$ indicates the number of items to be removed from the test after ith failure time. Progressive Type-II censored sample(T2CS) can be obtained according to pre-defined censoring scheme that describes the number of failure times $(\mathrm{m})$ with $\mathrm{n}-\mathrm{m}$ censored from $\mathrm{n}$ units.

The books dedicated to progressive censoring by [1] and [2] are basic sources including inference under progressive Type-I and Type-II censoring. [3] introduced progressively Type-I interval censoring by combining interval censoring and progressive censoring on Type-I censored data. [4] suggested conditional method for deriving exact confidence intervals for location, scale and quantiles when data from Type II progressive censored samples. The application of suggested method given on one and two parameter exponential models. [5] suggested approximate solution of parameters of Gaussian distributed Type-II censored samples. Approximate solution is used as a starting value of iterative solution of the likelihood equations.

Lots of works especially focused on the Weibull (WE) distribution parameter estimations are attracts the attention. [6] have discussed the Bayes estimates of WE distribution parameters under three different loss functions for progressive censored data. [7] have compared performance of the least square regression estimator and maximum likelihood estimation (MLE) for modified WE distribution. [8] have discussed parameter estimation of progressively censored random removed samples from WE distribution. [9] have derived maximum likelihood (ML) estimators for parameters of WE distribution based on progressive T2CS(s). [10] have derived parameter estimates of WE and Lognormal distribution based on progressive T2CS(s) using EM algorithm. [11] Discussed the ML estimates and Bayes estimates of WE distribution based on adaptive Type-II progressive hybrid censoring. [12] have developed a nonBayesian two-sample prediction based on a progressive Type-II right censoring scheme and obtained ML prediction for WE distribution. [13] proposed the use progressive external censoring at each stress level where the Type-II censoring is a special case, also obtained the ML estimates for WE regression parameters. [14] have obtained the unknown parameters of two-parameter inverse WE distribution based on progressive T2CS(s). [15] have obtained the MLEs of two-parameter exponentiated WE distribution under adaptive progressive T2CS(s) .

Most of the works on MLE of WE distribution parameters used newton method for solving the equation system obtained by partial derivatives of likelihood function according to distribution parameters. Even though it is popular, the biggest disadvantage of the Newton method is that it is a gradient based search algorithm which searches the optimum values of parameters depending on the inverse of the hessian matrix so it is valid only for functions that differentiable at least two times. Since the likelihood function is in more complex form for censored samples than in full samples, calculations of derivatives and related processes are more complicated. In this study we suggested the Genetic Algorithm (GA) which is derivative-free random search algorithm to MLE of T2CS for WE distribution. Although there are many applications of GA on different areas. There is no study such as the parameter estimation of WE distribution in T2CS with GA in the literature view. The importance of the work is in being the first work in literature which uses GA for parameter estimation in WE distributed T2CS(s).

\section{WEIBULL DISTRIBUTION}

WE distribution is one of the most widely used distribution bearing the name from Waloddi Weibull has a lot of applications in engineering and especially life time experiments, in terms of versatility and relative simplicity [16]. Probability density and distribution functions of the two-parameter WE are respectively has the form:

$f(x, \alpha, \beta)=\left\{\begin{array}{cc}\frac{\beta}{\alpha}\left(\frac{x}{\alpha}\right)^{\beta-1} e^{-\left(\frac{x}{\alpha}\right)^{\beta},}, \quad x>0 \\ 0, & \text { elsewhere }\end{array}\right.$

and

$F(x)=1-e^{-\left(\frac{x}{\alpha}\right)^{\beta}}$

where $\alpha$ and $\beta$ are positive scale and shape parameters respectively.

Let $x_{1: m: n}, x_{2: m: n}, \ldots, x_{m-1: m: n}, x_{m: m: n}$ be progressive T2CS from a two-parameter Weibull distribution, with censoring scheme

$\underline{r}=\left(r_{1}, r_{2} \cdots r_{m}\right)$.

The Likelihood function is given by 
$L(\alpha, \beta)=c\left(\frac{\beta}{\alpha}\right)^{m} \prod_{i=1}^{m}\left(\frac{x_{i: m: n}}{\alpha}\right)^{\beta-1} e^{-\sum_{i=1}^{m}\left(r_{i}+1\right)\left(\frac{x_{i: m: n}}{\alpha}\right)^{\beta}}$

where

$c=n\left(n-1-r_{1}\right)\left(n-2-r_{1}-r_{2}\right) \cdots$

$\times\left(n-(m-1)-r_{1}-r_{2}-\cdots-r_{m-1}\right)$

The Log likelihood function can be written as

$\log L(\alpha, \beta)=c \log (\beta)-m \beta \log (\alpha)+\cdots$

$+(\beta-1) \sum_{i=1}^{m} \log \left(x_{i}\right)-\sum_{i=1}^{m}\left(r_{i}+1\right)\left(\frac{x_{i}}{\alpha}\right)^{\beta}$

and hence the likelihood equations for $\alpha$ and $\beta$ are

$\frac{\partial \log L(\alpha, \beta)}{\partial \alpha}=-\frac{m \beta}{\alpha}+\beta \alpha^{-(\beta+1)} \sum_{i=1}^{m}\left(r_{i}+1\right)\left(x_{i}\right)^{\beta}=0$

and

$\frac{\partial \log L(\alpha, \beta)}{\partial \beta}=\frac{m}{\beta}-m \log (\alpha)+\sum_{i=1}^{m} \log \left(x_{i}\right) \cdots$

$-\sum_{i=1}^{m}\left(r_{i}+1\right)\left(\frac{x_{i}}{\alpha}\right)^{\beta} \log \left(\frac{x_{i}}{\alpha}\right)=0$

The MLEs of $\hat{\alpha}$ and $\hat{\beta}$ can be obtained by solving the equations (6) and (7). Equation (6) yields the MLE of $\alpha$ to be

$\hat{\alpha}=\left\{\frac{1}{m} \sum_{i=1}^{m}\left(r_{i}+1\right) x_{i}^{\widehat{\beta}}\right\}^{1 / \beta}$

Equation (7), in conjunction with the MLE of $\alpha$ in (8), reduces to

$\frac{1}{\widehat{\beta}}+\frac{1}{m} \sum_{i=1}^{m} \log \left(x_{i}\right)-\frac{\sum_{i=1}^{m}\left(r_{i}+1\right) x_{i}{ }^{\widehat{\beta}} \log \left(x_{i}\right)}{\sum_{i=1}^{m}\left(r_{i}+1\right) x_{i}{ }^{\widehat{\beta}}}=0$

Since (9) can't be solved analytically for $\hat{\beta}$, numerical methods can be employed such as Newton or etc.

\section{QUASI-NEWTON ALGORITHM}

Quasi newton algorithm is one type of learning algorithm that searches the global minimum of the objective function using quadratic approximation for unconstrained nonlinear numerical optimization problems.

Some features of the method may restrict the user. In order to be able to use the quasi-newton algorithm, the objective function must be differentiable at least twice. Quasi-newton uses gradient of objective function for estimating the hessian along the Newton Raphson direction $-H^{-1} \operatorname{grad}(f(\underline{x}))$. There are numerous Quasi Newton methods differs for the way that which approximation used for calculation in inverse of Hessian matrix. Detailed information about the approximations can be found from the studies of [22-24].

Quasi Newton steps for search the minimum of twice differentiable objective function $f(\underline{x})$ can be given briefly as follows:

Step 1: Define $\underline{x}_{0}$ starting point, set $\mathrm{k}=0$

Step 2: Estimate the $H^{-1}$ and calculate the search direction Step 3: Calculate the new point by taking $\underline{x}_{k+1}=\underline{x}_{k}-$ $\left[H^{-1}\right]_{k} * \operatorname{grad}\left(f\left(\underline{x}_{k}\right)\right)$

Step 4: Check the convergence by selected criteria (especially gradient).

Step 5: let $\mathrm{k}=\mathrm{k}+1$ and repeat from step 2 till convergence satisfies.

\section{GENETIC ALGORITHM}

GA is an evolutionary random search algorithm. GA has wide applications on different branches and first introduced by Holland in early 1960s and later developed by his student Goldberg. GAs are randomized search algorithms and are separated from classical optimization algorithms in terms of some features. These can be summarized as follows [17]:

GA uses the coding of the solutions instead of the solution values. GA searches for a set of points called population instead of points at each iteration to achieve the optimal solution.GA evaluates only the objective function to achieve the optimal solution and does not need another auxiliary knowledge such as derivatives of the objective function [18]. Thanks to this feature, it can be used to find the optimal solution of the non-derivative objective functions

GA uses probabilistic transition rules instead of deterministic rules. At each iteration every candidate solution in the population represented by chromosome. GA aims to achieve the optimal solution by applying genetic operators to chromosomes and evaluates them by the fitness (objective) function.

Because GA is inspired by natural selection and genetic principles, it has genetic operators called crossover, mutation and selection. Crossover and Mutation operators can be applied in a GA as follows:

Crossover operation to be applied a chromosome defined by the pre-defined crossover rate $(\mathrm{Cr})$. Let be the randomly generated crossover point indicator randomly generated from Bernoulli(Cr) is $\left[\begin{array}{llll}0 & 1 & 0 & 1\end{array}\right]$ where 1 selects gene from parent 1,0 select genes from parent 2 . For example let the

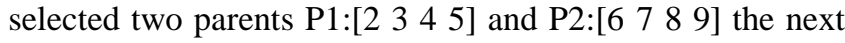
generation $\mathrm{C} 1$ of these parents can be obtained as $\mathrm{C} 1:\left[\begin{array}{ll}6 & 3\end{array}\right.$ 5] using crossover operator. This is called scattered crossover.

Mutation operator can be applied to a chromosome in two steps. At first step generate a random number according to 
pre-defined mutation rate $(\mathrm{Mr})$ from Bernoulli(Mr) for each gene in a chromosome. At the second step " 1 " values of generated number indicates the mutation of the gene by randomly regenerating it. Otherwise no change in gene. This type of mutation operator called uniform mutation.

GA Steps for parameter estimation of progressively T2CS from WE is as follows:

Let $\quad x_{1: m: n}, x_{2: m: n}, \cdots, x_{m-1: m: n}, x_{m: m: n}$ be Type-2 progressively censored sample from a two-parameter WE distribution with censoring scheme

$\underline{r}=\left(r_{1}, r_{2} \cdots r_{m}\right)$ and $\mathrm{c}$ given by in eq.(4).

Step 1: Define population size(N), select the coding type as real coding. Set roulette wheel selection which gives high fitness valued chromosomes more chance for selection to the next generation as selection method. Crossover method be the scattered crossover. Mutation method be uniform mutation. Use elitist strategy.

Step 2: Let $\theta_{i}=\left(\begin{array}{ll}\alpha_{i} & \beta_{i}\end{array}\right)$ are positive valued chromosomes which denotes the ith individual in a population as WE distribution parameter estimates denoted by genes $\alpha_{i}$ and $\beta_{i}$ $(\mathrm{i}=1,2 \cdots \mathrm{N})$. Randomly generate chromosomes $\mathrm{N}$ times and obtain $\mathrm{Nx} 2$ size initial population.

Step 3: Evaluate fitness of each chromosome by negative signed value of log likelihood function given in Eq.(5). Select the minimum fitness valued chromosome as elite chromosome and solution of problem as best fitness.

Step 4: If the first run of program go to step 5 else check the termination criteria (The difference of less than $\varepsilon=0.001$ between consecutive iterations in best fitness). If the criterium meets, go to step 9 otherwise go to step 5 .

Step 5: Select parents for next generation using selection method.

Step 6: Apply selected Crossover operator for diversity in population.

Step 7: Use Mutation operator for avoid to local optimum problem.

Step 8: Go to step 3

Step 9: Set best chromosome as solution.

\section{SIMULATION}

A Monte Carlo simulation study is conducted to compare the performance of Newton and GA on MLEs of Weibull distribution parameters. Matlab R2015a used for simulation study. Simulation algorithm for GA coded by user in Matlab according to section 4 . Newton estimates were obtained by using fminunc function by taking the starting values [0.01 0.01 ] and the objective function given in eq (5).

The progressive T2CS(s) are generated by using the algorithm suggested by [19]. We consider the scale and shape parameter setting respectively $(\alpha=1, \alpha=2)$ and ( $=0.5, \beta=1, \beta=2$ ) for different values of sample size(n) and number of failures (m) according to five different censoring schemes which adopted from the study of [20].

The proposed GA for parameter estimation of progressively T2CS from WE is defined as follows:

Population size(N) for each iteration set to 300. Scattered Crossover method selected in order to ensure the diversity in search space with the cross over probability by taking $\mathrm{Cr}=0.90$. Uniform mutation method selected for not to fall in to risk of local optimum by taking mutation probability $\mathrm{Mr}=0.01$. Elitist strategy used for saving best solution at each iteration for the next generation. WE distribution parameters $\theta=\left(\begin{array}{ll}\alpha & \beta\end{array}\right)$ are coded using real coding. It is also called chromosomes and contains randomly generated real valued two component which indicates the WE distribution parameter estimates for each individual in population. Negative signed value of $\mathrm{Eq}(5)$ is used for evaluating the fitness of chromosomes. The chromosome with best fitness value at each iteration selected as elite chromosome and kept for next generation. Parent selection for next generation applied using Roulette Wheel Selection which gives high fitness valued chromosomes more chance for selection to the next generation. The difference of less than $\varepsilon=0.001$ between consecutive iterations in fitness function is determined as the termination criterion. The best fitness valued chromosome at last generation gives the parameter estimates of WE distribution.

All process replicated 1000 times. To assess the performance of the GA and Newton, the simulated bias(Bias) and mean square error(MSE) values given by eq. (10) and eq. (11) are used. Significant differences between the methods are determined by the Wilcoxon signed rank test which results are given in Table 7.

$\operatorname{Bias}(\widehat{\theta})=\frac{\sum_{i=1}^{1000}\left(\widehat{\theta}_{i}-\theta\right)}{1000}$

and

$\operatorname{MSE}(\hat{\theta})=\frac{\sum_{i=1}^{1000}\left(\widehat{\theta}_{i}-\theta\right)^{2}}{1000}$ 
Table 1. Bias and MSE values of scale and shape parameters $(\alpha=1, \beta=0.5)$

\begin{tabular}{|c|c|c|c|c|c|c|c|c|c|c|c|}
\hline \multirow[t]{2}{*}{ State } & \multirow[t]{2}{*}{$\mathrm{N}$} & \multirow[t]{2}{*}{$\mathrm{m}$} & \multirow[t]{2}{*}{ Scheme } & \multicolumn{2}{|c|}{$\begin{array}{l}\text { Simulated } \\
\text { Biases }(\hat{\alpha})\end{array}$} & \multicolumn{2}{|c|}{$\begin{array}{l}\text { Simulated } \\
\text { Biases }(\hat{\beta}) \\
\end{array}$} & \multicolumn{2}{|c|}{$\operatorname{MSE}(\hat{\alpha})$} & \multicolumn{2}{|c|}{$\operatorname{MSE}(\hat{\beta})$} \\
\hline & & & & GA & Newton & GA & Newton & GA & Newton & GA & Newton \\
\hline 1 & 20 & 6 & 1 & 0.146 & -0.677 & -0.348 & -0.236 & 0.702 & 9.608 & 0.174 & 0.124 \\
\hline 2 & 20 & 6 & 2 & 0.326 & -0.276 & -0.070 & 0.008 & 0.494 & 0.494 & 0.083 & 0.085 \\
\hline 3 & 20 & 6 & 3 & 0.290 & -0.092 & -0.248 & -1.309 & 0.349 & 0.660 & 1.667 & 1.826 \\
\hline 4 & 20 & 6 & 4 & 0.154 & -0.825 & -0.277 & -0.127 & 0.467 & 1.066 & 0.146 & 0.094 \\
\hline 5 & 20 & 6 & 5 & 0.156 & -0.902 & -0.318 & -0.189 & 0.556 & 0.885 & 0.163 & 0.106 \\
\hline 6 & 20 & 12 & 1 & -0.035 & -0.922 & -0.211 & -0.277 & 0.746 & 0.896 & 0.156 & 0.129 \\
\hline 7 & 20 & 12 & 2 & 0.153 & -0.540 & -0.076 & -0.108 & 0.478 & 0.912 & 0.098 & 0.068 \\
\hline 8 & 20 & 12 & 3 & 0.111 & -0.917 & -0.247 & -0.249 & 0.620 & 0.907 & 0.160 & 0.102 \\
\hline 9 & 20 & 12 & 4 & 0.132 & -0.839 & -0.217 & -0.211 & 0.539 & 0.952 & 0.147 & 0.088 \\
\hline 10 & 20 & 12 & 5 & 0.103 & -0.900 & -0.251 & -0.230 & 0.615 & 0.873 & 0.154 & 0.099 \\
\hline 11 & 60 & 18 & 1 & -0.376 & 0.037 & -0.137 & -0.145 & 0.865 & 13.825 & 0.121 & 0.109 \\
\hline 12 & 60 & 18 & 2 & -0.214 & -0.840 & -0.178 & -0.127 & 0.310 & 0.875 & 0.107 & 0.043 \\
\hline 13 & 60 & 18 & 3 & -0.172 & -0.962 & -0.274 & -0.387 & 0.665 & 0.946 & 0.171 & 0.175 \\
\hline 14 & 60 & 18 & 4 & -0.182 & -0.974 & -0.312 & -0.320 & 0.438 & 0.950 & 0.175 & 0.125 \\
\hline 15 & 60 & 18 & 5 & -0.178 & -0.965 & -0.277 & -0.368 & 0.659 & 0.945 & 0.174 & 0.167 \\
\hline 16 & 60 & 36 & 1 & -0.532 & -0.048 & 0.003 & 0.083 & 0.907 & 6.405 & 0.105 & 0.088 \\
\hline 17 & 60 & 36 & 2 & -0.218 & -0.974 & -0.210 & -0.239 & 0.379 & 0.951 & 0.149 & 0.077 \\
\hline 18 & 60 & 36 & 3 & -0.283 & -0.914 & -0.104 & -0.396 & 0.805 & 0.883 & 0.147 & 0.183 \\
\hline 19 & 60 & 36 & 4 & -0.234 & -0.986 & -0.181 & -0.393 & 0.605 & 0.974 & 0.172 & 0.169 \\
\hline 20 & 60 & 36 & 5 & -0.268 & -0.981 & -0.095 & -0.384 & 0.722 & 0.966 & 0.157 & 0.168 \\
\hline
\end{tabular}

Table 2. Bias and MSE values of scale and shape parameters $(\alpha=1, \beta=1)$

\begin{tabular}{|c|c|c|c|c|c|c|c|c|c|c|c|}
\hline \multirow[t]{2}{*}{ State } & \multirow[t]{2}{*}{$\mathrm{N}$} & \multirow[t]{2}{*}{$\mathrm{m}$} & \multirow[t]{2}{*}{ Scheme } & \multicolumn{2}{|c|}{$\begin{array}{l}\text { Simulated Biases } \\
(\hat{\alpha})\end{array}$} & \multicolumn{2}{|c|}{$\begin{array}{l}\text { Simulated Biases } \\
(\hat{\beta})\end{array}$} & \multicolumn{2}{|c|}{$\operatorname{MSE}(\hat{\alpha})$} & \multicolumn{2}{|c|}{$\operatorname{MSE}(\hat{\beta})$} \\
\hline & & & & GA & Newton & GA & Newton & GA & Newton & GA & Newton \\
\hline 21 & 20 & 6 & 1 & 0.025 & -0.748 & -0.422 & -0.616 & 0.397 & 0.661 & 0.579 & 0.417 \\
\hline 22 & 20 & 6 & 2 & 0.419 & -0.297 & 0.048 & -0.006 & 0.342 & 0.374 & 0.418 & 0.232 \\
\hline 23 & 20 & 6 & 3 & 0.109 & -0.695 & -0.331 & -0.468 & 0.377 & 0.632 & 0.499 & 0.263 \\
\hline 24 & 20 & 6 & 4 & 0.244 & -0.547 & -0.076 & -0.328 & 0.358 & 0.510 & 0.438 & 0.220 \\
\hline 25 & 20 & 6 & 5 & 0.146 & -0.649 & -0.178 & -0.455 & 0.377 & 0.657 & 0.484 & 0.251 \\
\hline 26 & 20 & 12 & 1 & -0.087 & -0.883 & -0.558 & -0.567 & 0.486 & 0.801 & 0.642 & 0.358 \\
\hline 27 & 20 & 12 & 2 & 0.089 & -0.616 & -0.155 & -0.176 & 0.311 & 0.512 & 0.399 & 0.220 \\
\hline 28 & 20 & 12 & 3 & 0.024 & -0.867 & -0.547 & -0.580 & 0.443 & 0.771 & 0.590 & 0.350 \\
\hline 29 & 20 & 12 & 4 & 0.142 & -0.772 & -0.344 & -0.480 & 0.370 & 0.636 & 0.517 & 0.272 \\
\hline 30 & 20 & 12 & 5 & 0.081 & -0.835 & -0.476 & -0.554 & 0.414 & 0.743 & 0.567 & 0.327 \\
\hline 31 & 60 & 18 & 1 & -0.415 & -0.933 & -0.830 & -0.793 & 0.417 & 0.872 & 0.738 & 0.633 \\
\hline 32 & 60 & 18 & 2 & -0.336 & -0.716 & -0.563 & -0.222 & 0.284 & 0.564 & 0.373 & 0.216 \\
\hline 33 & 60 & 18 & 3 & -0.397 & -0.908 & -0.783 & -0.676 & 0.367 & 0.828 & 0.664 & 0.458 \\
\hline 34 & 60 & 18 & 4 & -0.447 & -0.775 & -0.629 & -0.607 & 0.316 & 0.628 & 0.465 & 0.396 \\
\hline 35 & 60 & 18 & 5 & -0.416 & -0.871 & -0.764 & -0.666 & 0.357 & 0.764 & 0.624 & 0.445 \\
\hline 36 & 60 & 36 & 1 & -0.340 & -0.988 & -0.683 & -0.923 & 0.649 & 0.977 & 0.604 & 0.863 \\
\hline 37 & 60 & 36 & 2 & -0.260 & -0.914 & -0.767 & -0.520 & 0.301 & 0.840 & 0.624 & 0.306 \\
\hline 38 & 60 & 36 & 3 & -0.216 & -0.983 & -0.833 & -0.783 & 0.448 & 0.966 & 0.760 & 0.645 \\
\hline 39 & 60 & 36 & 4 & -0.312 & -0.938 & -0.825 & -0.715 & 0.341 & 0.880 & 0.720 & 0.519 \\
\hline 40 & 60 & 36 & 5 & -0.230 & -0.982 & -0.815 & -0.784 & 0.460 & 0.964 & 0.738 & 0.621 \\
\hline
\end{tabular}


Table 3. Bias and MSE values of scale and shape parameters $(\alpha=1, \beta=2)$

\begin{tabular}{|l|l|l|l|r|r|r|r|r|r|r|r|}
\hline \multirow{2}{*}{ State } & \multirow{2}{*}{$\mathrm{N}$} & \multirow{2}{*}{$\mathrm{m}$} & \multirow{2}{*}{ Scheme } & \multicolumn{2}{|c|}{\begin{tabular}{c} 
Simulated Biases \\
\cline { 5 - 12 }
\end{tabular}} & & & & \multicolumn{2}{|c|}{ Simulated Biases } & \multicolumn{2}{|c|}{ MSE $(\hat{\alpha})$} & \multicolumn{2}{|c|}{ MSE $(\hat{\beta})$} \\
\hline & & & GA & Newton & GA & Newton & GA & Newton & GA & Newton \\
\hline 41 & 20 & 6 & 1 & 0.232 & -0.221 & -0.195 & -1.349 & 0.337 & 0.431 & 1.848 & 2.029 \\
\hline 42 & 20 & 6 & 2 & 0.444 & 0.169 & -0.152 & -0.759 & 0.347 & 1.719 & 0.521 & 0.980 \\
\hline 43 & 20 & 6 & 3 & 0.285 & -0.041 & -0.157 & -1.279 & 0.332 & 0.792 & 1.710 & 1.781 \\
\hline 44 & 20 & 6 & 4 & 0.346 & 0.332 & -0.093 & -1.082 & 0.328 & 0.861 & 1.343 & 1.364 \\
\hline 45 & 20 & 6 & 5 & 0.298 & 0.111 & -0.159 & -1.212 & 0.338 & 0.967 & 1.558 & 1.668 \\
\hline 46 & 20 & 12 & 1 & 0.160 & -0.775 & -0.634 & -1.556 & 0.431 & 0.648 & 2.510 & 2.425 \\
\hline 47 & 20 & 12 & 2 & 0.269 & -0.604 & -0.345 & -1.252 & 0.343 & 0.423 & 1.881 & 1.706 \\
\hline 48 & 20 & 12 & 3 & 0.423 & 0.564 & 2.428 & 2.200 & 0.641 & 1.210 & 0.034 & 0.655 \\
\hline 49 & 20 & 12 & 4 & 0.272 & -0.631 & -0.437 & -1.349 & 0.395 & 0.427 & 2.068 & 1.849 \\
\hline 50 & 20 & 12 & 5 & 0.265 & -0.701 & -0.629 & -1.444 & 0.410 & 0.502 & 2.296 & 2.091 \\
\hline 51 & 60 & 18 & 1 & -0.481 & -0.652 & -1.634 & -1.708 & 0.316 & 0.441 & 2.727 & 2.919 \\
\hline 52 & 60 & 18 & 2 & -0.274 & -0.493 & -1.362 & -1.232 & 0.189 & 0.436 & 1.889 & 1.742 \\
\hline 53 & 60 & 18 & 3 & -0.450 & -0.637 & -1.564 & -1.550 & 0.292 & 0.543 & 2.506 & 2.479 \\
\hline 54 & 60 & 18 & 4 & -0.310 & -0.104 & -1.392 & -1.176 & 0.152 & 0.683 & 1.987 & 1.850 \\
\hline 55 & 60 & 18 & 5 & -0.390 & -0.441 & -1.503 & -1.451 & 0.225 & 0.560 & 2.318 & 2.311 \\
\hline 56 & 60 & 36 & 1 & -0.334 & -0.941 & -1.828 & -1.738 & 0.401 & 0.887 & 3.399 & 3.021 \\
\hline 57 & 60 & 36 & 2 & -0.356 & -0.820 & -1.641 & -1.542 & 0.302 & 0.676 & 2.726 & 2.380 \\
\hline 58 & 60 & 36 & 3 & -0.380 & -0.919 & -1.795 & -1.718 & 0.366 & 0.845 & 3.269 & 2.954 \\
\hline 59 & 60 & 36 & 4 & -0.440 & -0.790 & -1.683 & -1.587 & 0.326 & 0.628 & 2.877 & 2.523 \\
\hline 60 & 60 & 36 & 5 & -0.311 & -0.905 & -1.810 & -1.602 & 0.347 & 0.821 & 3.315 & 2.579 \\
\hline
\end{tabular}

Table 4. Bias and MSE values of scale and shape parameters $(\alpha=2, \beta=0.5)$

\begin{tabular}{|l|l|l|l|r|r|r|r|r|r|r|r|}
\hline \multirow{2}{*}{ State } & \multirow{2}{*}{$\mathrm{N}$} & \multirow{2}{*}{$\mathrm{m}$} & \multirow{2}{*}{ Scheme } & \multicolumn{2}{|c|}{\begin{tabular}{c} 
Simulated Biases \\
\cline { 5 - 12 }
\end{tabular}} & & & & \multicolumn{2}{|c|}{ Simulated Biases } & \multicolumn{2}{|c|}{ MSE $(\hat{\alpha})$} & \multicolumn{2}{|c|}{ MSE $(\hat{\beta})$} \\
\hline 61 & 20 & 6 & 1 & -1.116 & -1.795 & -0.260 & -0.229 & 2.388 & 3.702 & 0.140 & 0.097 \\
\hline 62 & 20 & 6 & 2 & -0.064 & -0.068 & 0.001 & 0.046 & 1.510 & 4.475 & 0.051 & 0.061 \\
\hline 63 & 20 & 6 & 3 & -1.076 & -1.977 & -0.133 & -0.297 & 2.567 & 3.912 & 0.157 & 0.109 \\
\hline 64 & 20 & 6 & 4 & -1.055 & -1.367 & -0.121 & -0.007 & 1.970 & 4.609 & 0.098 & 0.080 \\
\hline 65 & 20 & 6 & 5 & -0.871 & -0.581 & -0.208 & -0.256 & 1.317 & 2.163 & 0.182 & 0.190 \\
\hline 66 & 20 & 12 & 1 & -0.976 & -1.759 & -0.187 & -0.191 & 2.231 & 3.550 & 0.137 & 0.128 \\
\hline 67 & 20 & 12 & 2 & -0.191 & -0.313 & -0.021 & -0.004 & 1.523 & 2.855 & 0.048 & 0.038 \\
\hline 68 & 20 & 12 & 3 & -0.936 & -1.350 & -0.150 & -0.080 & 2.017 & 3.422 & 0.106 & 0.082 \\
\hline 69 & 20 & 12 & 4 & -0.724 & -1.067 & -0.116 & -0.046 & 1.815 & 3.220 & 0.086 & 0.048 \\
\hline 70 & 20 & 12 & 5 & -0.957 & -1.377 & -0.141 & -0.095 & 1.942 & 3.267 & 0.101 & 0.062 \\
\hline 71 & 60 & 18 & 1 & -1.095 & -1.550 & -0.189 & -0.325 & 2.912 & 7.204 & 0.147 & 0.170 \\
\hline 72 & 60 & 18 & 2 & -0.690 & -0.973 & -0.077 & -0.062 & 1.765 & 3.090 & 0.054 & 0.027 \\
\hline 73 & 60 & 18 & 3 & -1.090 & -1.955 & -0.284 & -0.262 & 2.193 & 3.840 & 0.165 & 0.090 \\
\hline 74 & 60 & 18 & 4 & -1.293 & -1.888 & -0.237 & -0.168 & 1.976 & 3.596 & 0.136 & 0.052 \\
\hline 75 & 60 & 18 & 5 & -1.146 & -1.943 & -0.290 & -0.228 & 2.213 & 3.789 & 0.164 & 0.072 \\
\hline 76 & 60 & 36 & 1 & -1.212 & -1.256 & 0.008 & -0.311 & 3.394 & 7.679 & 0.116 & 0.160 \\
\hline 77 & 60 & 36 & 2 & -1.087 & -1.782 & -0.170 & -0.178 & 1.643 & 3.460 & 0.096 & 0.049 \\
\hline 78 & 60 & 36 & 3 & -1.076 & -1.977 & -0.133 & -0.297 & 2.567 & 3.912 & 0.157 & 0.109 \\
\hline 79 & 60 & 36 & 4 & -1.177 & -1.964 & -0.210 & -0.264 & 1.982 & 3.861 & 0.163 & 0.082 \\
\hline 80 & 60 & 36 & 5 & -1.103 & -1.973 & -0.133 & -0.265 & 2.281 & 3.896 & 0.155 & 0.085 \\
\hline
\end{tabular}


Table 5. Bias and MSE values of scale and shape parameters $(\alpha=2, \beta=1)$

\begin{tabular}{|c|c|c|c|c|c|c|c|c|c|c|c|}
\hline \multirow[t]{2}{*}{ State } & \multirow[t]{2}{*}{$\mathrm{N}$} & \multirow[t]{2}{*}{$\mathrm{m}$} & \multirow[t]{2}{*}{ Scheme } & \multicolumn{2}{|c|}{$\begin{array}{l}\text { Simulated Biases } \\
(\hat{\alpha})\end{array}$} & \multicolumn{2}{|c|}{$\begin{array}{l}\text { Simulated Biases } \\
(\hat{\beta})\end{array}$} & \multicolumn{2}{|c|}{$\operatorname{MSE}(\hat{\alpha})$} & \multicolumn{2}{|c|}{$\operatorname{MSE}(\hat{\beta})$} \\
\hline & & & & GA & Newton & GA & Newton & GA & Newton & GA & Newton \\
\hline 81 & 20 & 6 & 1 & -1.195 & -1.236 & -0.387 & -0.438 & 1.645 & 2.016 & 0.273 & 0.328 \\
\hline 82 & 20 & 6 & 2 & -0.019 & -0.077 & 0.074 & 0.133 & 0.661 & 0.856 & 0.122 & 0.137 \\
\hline 83 & 20 & 6 & 3 & -1.399 & -1.835 & -0.750 & -0.598 & 2.157 & 3.391 & 0.632 & 0.415 \\
\hline 84 & 20 & 6 & 4 & -0.486 & -0.415 & -0.116 & -0.060 & 1.163 & 1.417 & 0.139 & 0.140 \\
\hline 85 & 20 & 6 & 5 & -0.875 & -0.558 & -0.226 & -0.268 & 1.313 & 2.173 & 0.191 & 0.184 \\
\hline 86 & 20 & 12 & 1 & -1.241 & -1.214 & -0.574 & -0.499 & 1.783 & 2.338 & 0.404 & 0.339 \\
\hline 87 & 20 & 12 & 2 & -0.252 & -0.217 & -0.087 & 0.160 & 0.833 & 0.851 & 0.113 & 0.224 \\
\hline 88 & 20 & 12 & 3 & -1.059 & -1.061 & -0.440 & -0.168 & 1.506 & 1.954 & 0.271 & 0.426 \\
\hline 89 & 20 & 12 & 4 & -0.680 & -0.724 & -0.264 & 0.010 & 1.218 & 1.461 & 0.162 & 0.211 \\
\hline 90 & 20 & 12 & 5 & -0.927 & -0.929 & -0.384 & -0.112 & 1.364 & 1.722 & 0.241 & 0.381 \\
\hline 91 & 60 & 18 & 1 & -1.447 & -1.590 & -0.678 & -0.740 & 2.209 & 2.812 & 0.525 & 0.551 \\
\hline 92 & 60 & 18 & 2 & -0.831 & -0.724 & -0.254 & 0.038 & 1.201 & 1.454 & 0.141 & 0.187 \\
\hline 93 & 60 & 18 & 3 & -1.427 & -1.650 & -0.596 & -0.541 & 2.133 & 2.838 & 0.423 & 0.351 \\
\hline 94 & 60 & 18 & 4 & -1.237 & -1.194 & -0.374 & -0.238 & 1.638 & 1.913 & 0.212 & 0.343 \\
\hline 95 & 60 & 18 & 5 & -1.375 & -1.482 & -0.528 & -0.490 & 1.976 & 2.474 & 0.342 & 0.367 \\
\hline 96 & 60 & 36 & 1 & -1.339 & -1.930 & -0.761 & -0.790 & 2.170 & 3.731 & 0.673 & 0.660 \\
\hline 97 & 60 & 36 & 2 & -1.321 & -1.434 & -0.524 & -0.166 & 1.872 & 2.246 & 0.331 & 0.171 \\
\hline 98 & 60 & 36 & 3 & -1.399 & -1.835 & -0.750 & -0.598 & 2.157 & 3.391 & 0.632 & 0.415 \\
\hline 99 & 60 & 36 & 4 & -1.401 & -1.513 & -0.624 & -0.600 & 2.083 & 3.828 & 0.456 & 0.382 \\
\hline 100 & 60 & 36 & 5 & -1.362 & -1.618 & -0.718 & -0.295 & 2.068 & 2.721 & 0.571 & 0.283 \\
\hline
\end{tabular}

Table 6. Bias and MSE values of scale and shape parameters $(\alpha=2, \beta=2)$

\begin{tabular}{|l|l|l|l|r|r|r|r|r|r|r|r|}
\hline \multirow{2}{*}{ State } & \multirow{2}{*}{$\mathrm{N}$} & \multirow{2}{*}{$\mathrm{m}$} & \multirow{2}{*}{ Scheme } & \multicolumn{2}{|c|}{\begin{tabular}{c} 
Simulated Biases \\
\cline { 5 - 12 }
\end{tabular}} & & & & \multicolumn{2}{c|}{ Simulated Biases } & \multicolumn{2}{|c|}{ MSE $(\hat{\alpha})$} & \multicolumn{2}{|c|}{ MSE $(\hat{\beta})$} \\
\hline 101 & 20 & 6 & 1 & -0.419 & -0.285 & -0.991 & -1.034 & 0.476 & 1.156 & 1.148 & 1.329 \\
\hline 102 & 20 & 6 & 2 & -0.021 & -0.001 & -0.058 & -0.121 & 0.179 & 0.185 & 0.233 & 0.236 \\
\hline 103 & 20 & 6 & 3 & -1.381 & -1.373 & -1.600 & -1.551 & 1.981 & 2.231 & 2.606 & 2.442 \\
\hline 104 & 20 & 6 & 4 & 0.114 & 0.511 & -0.467 & -0.623 & 0.412 & 0.838 & 0.436 & 0.618 \\
\hline 105 & 20 & 6 & 5 & -0.002 & 1.480 & -0.732 & -1.000 & 0.548 & 4.095 & 0.743 & 1.097 \\
\hline 106 & 20 & 12 & 1 & -0.972 & -0.855 & -1.326 & -1.460 & 1.171 & 3.115 & 1.802 & 2.194 \\
\hline 107 & 20 & 12 & 2 & -0.340 & -0.420 & -0.849 & -0.122 & 0.464 & 0.830 & 0.844 & 0.830 \\
\hline 108 & 20 & 12 & 3 & -0.780 & -0.887 & -1.209 & -0.830 & 0.950 & 1.249 & 1.520 & 1.117 \\
\hline 109 & 20 & 12 & 4 & -0.312 & -0.583 & -1.018 & -0.585 & 0.474 & 0.939 & 1.121 & 0.804 \\
\hline 110 & 20 & 12 & 5 & -0.598 & -0.802 & -1.154 & -0.803 & 0.739 & 1.052 & 1.386 & 0.978 \\
\hline 111 & 60 & 18 & 1 & -1.023 & -0.787 & -1.291 & -1.614 & 1.145 & 0.983 & 1.741 & 2.628 \\
\hline 112 & 60 & 18 & 2 & -0.491 & -0.630 & -1.016 & -0.786 & 0.636 & 1.065 & 1.101 & 1.077 \\
\hline 113 & 60 & 18 & 3 & -1.038 & -1.019 & -1.239 & -1.291 & 1.169 & 1.518 & 1.599 & 1.840 \\
\hline 114 & 60 & 18 & 4 & -0.490 & -0.469 & -1.042 & -0.998 & 0.499 & 0.938 & 1.186 & 1.278 \\
\hline 115 & 60 & 18 & 5 & -0.811 & -0.710 & -1.137 & -1.206 & 0.842 & 1.299 & 1.384 & 1.692 \\
\hline 116 & 60 & 36 & 1 & -1.455 & -1.688 & -1.671 & -1.737 & 2.188 & 2.857 & 2.852 & 3.021 \\
\hline 117 & 60 & 36 & 2 & -1.246 & -1.265 & -1.348 & -1.012 & 1.618 & 2.080 & 1.860 & 1.619 \\
\hline 118 & 60 & 36 & 3 & -1.381 & -1.373 & -1.600 & -1.551 & 1.981 & 2.231 & 2.606 & 2.442 \\
\hline 119 & 60 & 36 & 4 & 0.532 & -0.605 & -0.616 & -1.644 & 1.671 & 4.864 & 2.488 & 2.723 \\
\hline 120 & 60 & 36 & 5 & 0.373 & -1.228 & -0.717 & -1.679 & 1.732 & 1.620 & 2.632 & 2.828 \\
\hline
\end{tabular}


Table 7. MSE and Bias statistics for GA and Newton algorithms

\begin{tabular}{|l|c|c|c|c|c|c|c|c|}
\hline & \multicolumn{2}{|c|}{ Simulated Biases $(\hat{\alpha})$} & \multicolumn{2}{c|}{ Simulated Biases $(\hat{\beta})$} & \multicolumn{2}{c|}{ MSE $(\hat{\alpha})$} & \multicolumn{2}{c|}{ MSE $(\hat{\beta})$} \\
\hline & GA & Newton & GA & Newton & GA & Newton & GA & Newton \\
\hline Mean & -.466 & -.867 & -.535 & -.602 & 1.023 & 1.952 & .827 & .786 \\
\hline Std. Error of Mean & .051 & .054 & .051 & .054 & .069 & .179 & .082 & .081 \\
\hline Median & -.366 & -.877 & -.361 & -.474 & .654 & .975 & .447 & .346 \\
\hline P value (Wilcoxon) & \multicolumn{3}{|c|}{.000} & \multicolumn{2}{|c|}{.738} & \multicolumn{2}{c|}{.000} & \multicolumn{2}{c|}{.001} \\
\hline
\end{tabular}

Table 8. Censoring schemes used in the simulation study

\begin{tabular}{|c|c|}
\hline Scheme No. & $\left(\mathrm{R}_{1}, \mathrm{R}_{2}, \cdots \mathrm{R}_{\mathrm{m}-1}, \mathrm{R}_{\mathrm{m}}\right)$ \\
\hline 1 & $(0,0 \cdots 0, \mathrm{n}-\mathrm{m})$ \\
\hline 2 & $(\mathrm{n}-\mathrm{m}, 0,0 \cdots, 0)$ \\
\hline 3 & $\left(\frac{(n-m)}{2}, 0,0 \cdots 0, \frac{(n-m)}{2}\right)$ \\
\hline 4 & $\left(0,0 \cdots \frac{(n-m)}{2}, \frac{(n-m)}{2} \cdots 0,0\right)$ \\
\hline 5 & $\left(\sim \frac{(n-m)}{m}, \sim \frac{(n-m)}{m} \cdots \sim \frac{(n-m)}{m}, \sim \frac{(n-m)}{m}\right)$ \\
\hline
\end{tabular}

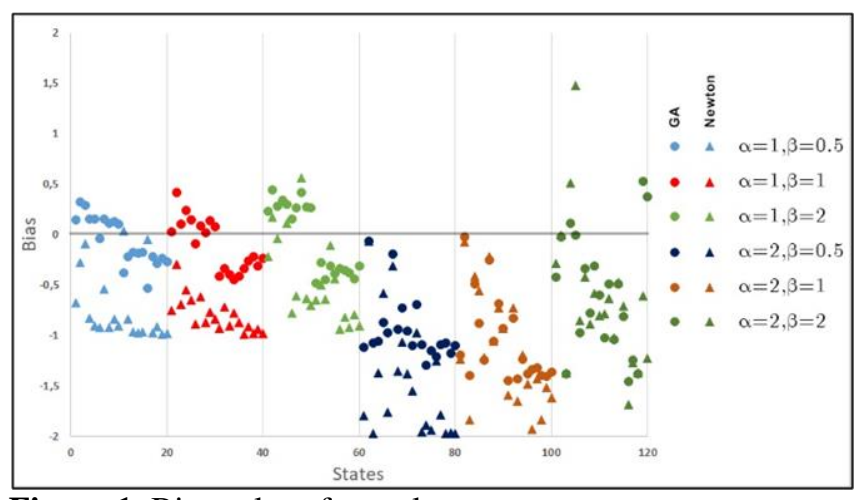

Figure 1. Bias values for scale parameter

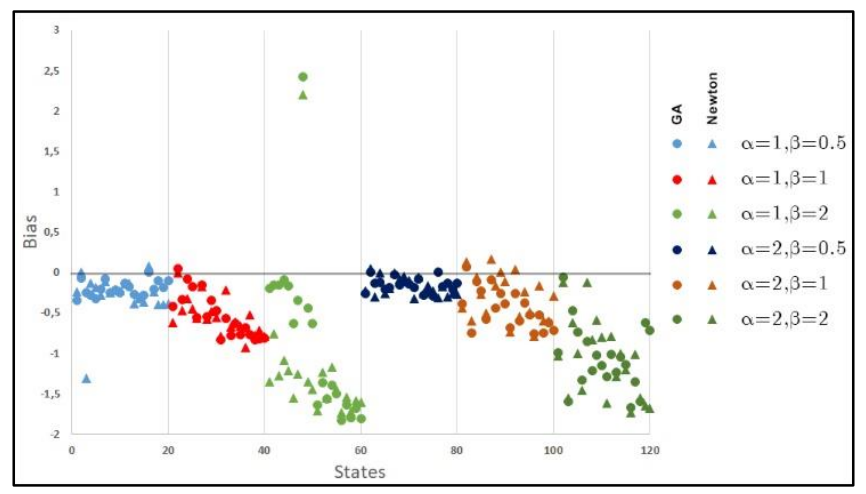

Figure 2. Bias values for shape parameter

The Figure 1-6 that represents the results of Table 1-6 can be briefly summarized as follows. According to the bias comparisons of the scale parameter given in Figure 1, difference between GA and Newton is found statistically significant at $5 \%$ significant level by Wilcoxon test $(p=.000<.05)$. The mean of simulated biases of GA is closer to zero than Newton, also has less variability as shown in Table 7. Shape parameter biases are quite similar for both methods as shown in Figure 2. Wilcoxon test results showed that shape parameter biases are found similar and there is no significance difference between two methods $(\mathrm{p}=.738>.05)$ at $5 \%$ significance level.

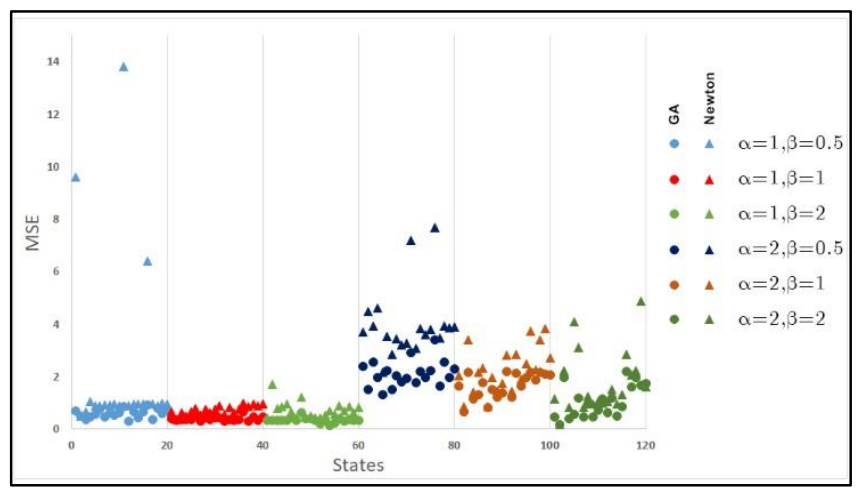

Figure 3. MSE values for scale parameter

According to the MSE comparisons of the scale parameter given in Figure 3, difference between GA and Newton is found statistically significant at 5\% significant level by Wilcoxon test $(\mathrm{p}=.000<.05)$. The mean of MSEs of GA is smaller than Newton, also has less variability as shown in Table 7. Although shape parameter MSEs looks quite similar for both methods as in Figure 4, Wilcoxon test results showed that shape parameter MSEs are found statistically different for two methods $(\mathrm{p}=.001<.05)$ at $5 \%$ significance level. Newton has smaller mean and less variability than GA for shape parameter MSEs.

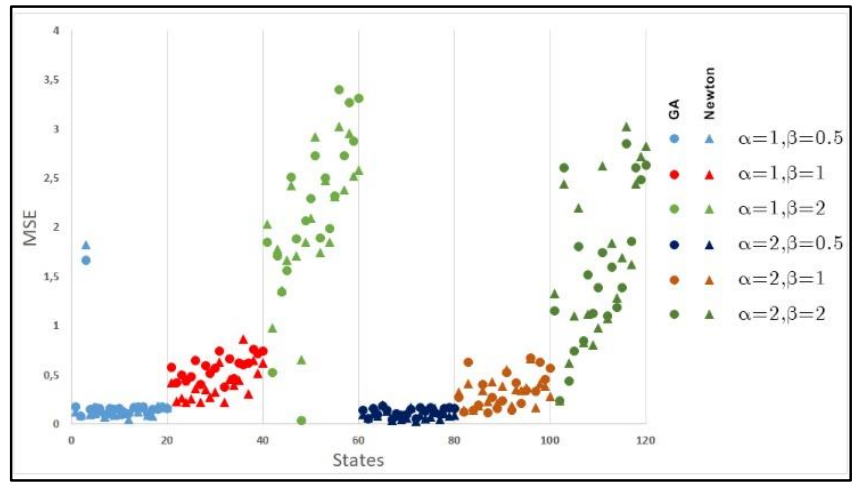

Figure 4. MSE values for shape parameter

Bias comparisons for different censoring schemes of scale and shape parameters according to Table 1-6 are also given in Figure 5-8. Wilcoxon test results for comparisons of methods for 5 different schemes are given in Table 9. 
Table 9. P values of Wilcoxon test for schemes

\begin{tabular}{|l|l|l|l|l|l|}
\hline Scheme & 1 & 2 & 3 & 4 & 5 \\
\hline $\begin{array}{l}\text { Simulated } \\
\text { Biases }(\hat{\alpha})\end{array}$ & .002 & .000 & .000 & .001 & .002 \\
\hline $\begin{array}{l}\text { Simulated } \\
\text { Biases }(\hat{\beta})\end{array}$ & .021 & .052 & .732 & .830 & .458 \\
\hline $\operatorname{MSE}(\hat{\alpha})$ & .000 & .000 & .000 & .000 & .000 \\
\hline $\operatorname{MSE}(\hat{\beta})$ & .607 & .011 & .056 & .040 & .153 \\
\hline
\end{tabular}

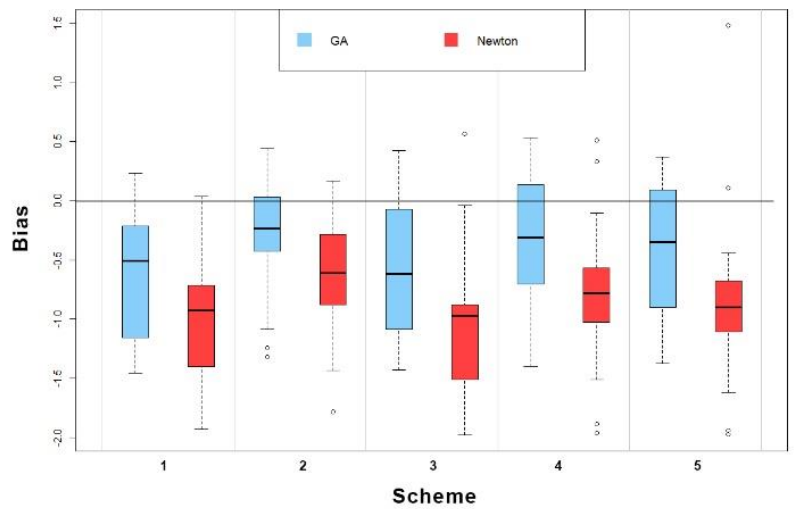

Figure 5. Bias values of scale parameters for different schemes

According to the bias comparisons of the scale parameter given in Figure 5, difference between GA and Newton is found statistically significant at 5\% significant level by Wilcoxon test for 5 different censoring schemes. (all $p$ values given at $2^{\text {nd }}$ row of Table 9 are smaller than .05) Bias means of GA are closer to zero than those Newton's for all schemes as seen in Figure 5.

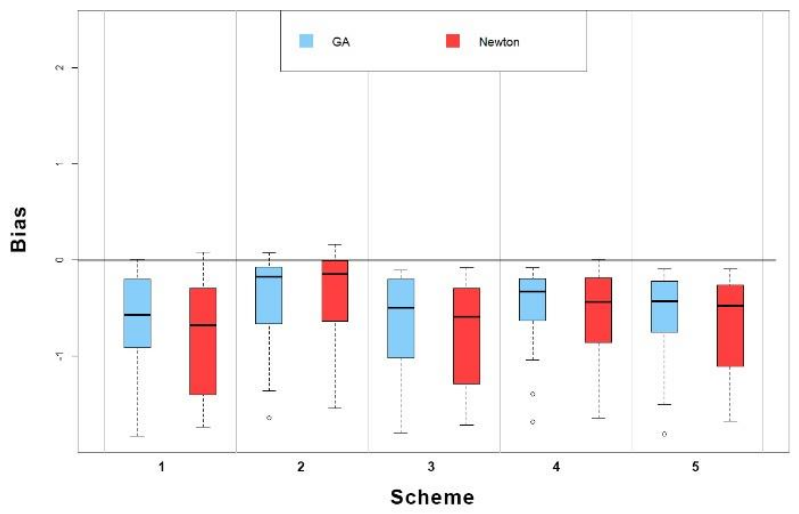

Figure 6. Bias values of shape parameters for different schemes

According to the bias comparisons of the shape parameter given in Figure 6, There is no significance difference between two methods except scheme 1 ( $\mathrm{p}$ values given at $3^{\text {rd }}$ row of Table 9$)(\mathrm{p}=.021<.05)$ at $5 \%$ significance level. Mean bias of GA is closer to zero than Newton's for scheme 1 as seen in Figure 6.

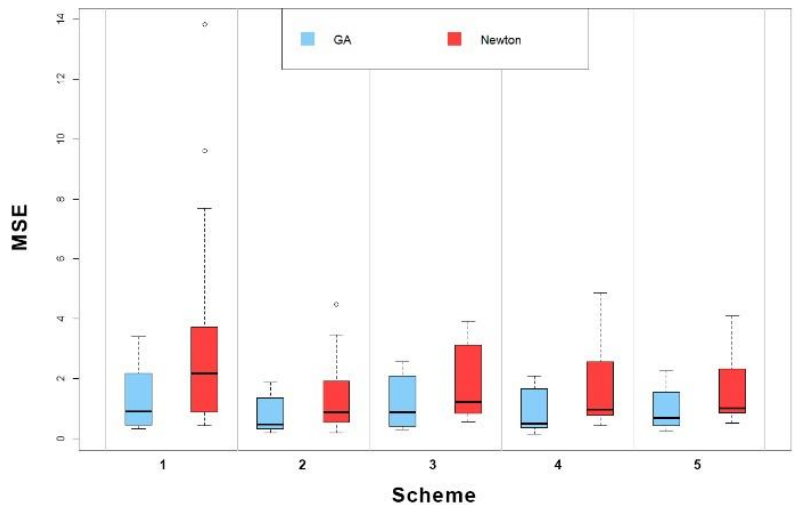

Figure 7. MSE values of scale parameters for different schemes

According to the MSE comparisons of the scale parameter given in Figure 7, difference between GA and Newton is found statistically significant at $5 \%$ significant level by Wilcoxon test for 5 different censoring schemes. ( $p$ values given in Table 9 row 4 are smaller than .05) GA's mean MSE values are smaller than those Newton's for all schemes as seen in Figure 7.

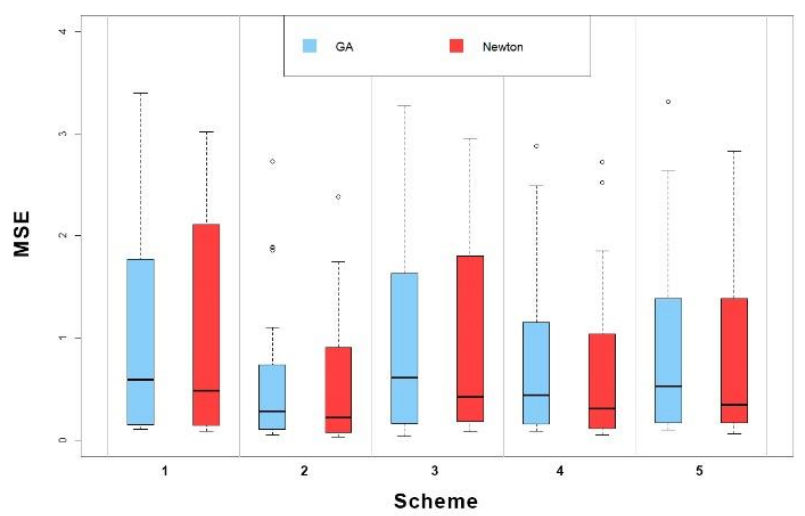

Figure 8. MSE values of shape parameters for different schemes

According to the MSE comparisons of the shape parameter given in Figure 8, difference between GA and Newton is found statistically significant at 5\% significant level by Wilcoxon test for schemes 2 and 4 . (p values given in $5^{\text {th }}$ row of Table 9) GA's mean MSE values are higher than those Newton's for all schemes in Figure 8.

\section{REAL DATA APPLICATION}

In this section, we consider the data set representing the time to deterioration of an insulating fluid between the electrodes at a voltage of $34 \mathrm{k} . \mathrm{v}$. [21] [1] The data set consist of $m=8$ observation from $\mathrm{n}=19$ unit with censoring scheme are $x_{8: 19}=(.19 .78 .961 .312 .784 .856 .507 .35)$ and $R=\left(\begin{array}{llllllll}0 & 0 & 3 & 0 & 3 & 0 & 0 & 5\end{array}\right)$.

We use Newton and GA method to obtain the point estimations of the two-parameter WE distribution based on progressive type 2 censoring scheme. 
Parameter estimates are found as $\left(\hat{\alpha}_{G A}=9.2253\right.$, $\left.\hat{\alpha}_{\text {Newton }}=9.2254, \quad \hat{\beta}_{G A}=0.9743, \quad \widehat{\beta}_{\text {Newton }}=0.9743\right)$. Scale and shape parameter estimates are similar and compliance with the results given in [21] for same data with $(\hat{\alpha}=9.2254, \hat{\beta}=0.9743)$.

\section{RESULTS AND CONCLUSION}

MLE is one of the most frequently used parameter estimation methods especially for censored samples. Newton is one of the widely used methods for solving the system of equations especially in maximum likelihood estimation. Even though it is popular, the biggest disadvantage of the Newton method is that it is a valid method for only differentiable functions. GA is very popular and derivative-free learning algorithm. In this study we suggest GA for MLE of progressive T2CS from WE distribution which has more complex likelihood function than full case.

According to simulation results we compared the bias and MSE performance of GA and Newton methods. As a result of study our findings are as follows: GA is better than Newton for scale parameter MLE in terms of Bias and MSE. There is no difference between GA and Newton for shape parameter biases of MLE. Newton is better than GA for shape parameter MSE of MLE.

GA is better than Newton for scale parameter biases and MSEs for all schemes. GA is better than Newton for Shape parameter biases for scheme1. There is no difference between GA and Newton in terms of shape parameter biases for schemes except scheme1.

In terms of shape parameter MSEs Newton is better than GA for scheme 2 and scheme 4 but there is no difference between methods for other schemes.

\section{REFERENCES}

[1]. N. Balakrishnan and R. Aggarwala, Progressive censoring: theory, methods, and applications, Springer Science \& Business Media, 2000.

[2]. N. Balakrishnan and C. Erhard, The art of progressive censoring, Springer New York, 2014.

[3]. R. Aggarwala, "Progressive interval censoring: some mathematical results with applications to inference," Communications in Statistics-Theory and Methods, vol. 39, no. 8-9, pp. 1921-1935, 2001.

[4]. R. Viveros and N. Balakrishnan, "Interval estimation of parameters of life from progressively censored data," Technometrics, vol. 36, no. 1, pp. 84-91, 1994.

[5]. N. Balakrishnan, N. Kannan and C. Lin, "Point and interval estimation for Gaussian distribution, based on progressively," IEEE Transactions on Reliability, vol. 52, no. 1, pp. 90-95, 2003.

[6]. R. R. Abu Awwad, M. Z. Raqap and M. A.-M. Intesar, "Statistical inference based on progressively type II censored data from Weibull model," Communications in StatisticsSimulation and Computation, vol. 44, no. 10, pp. 2654-2670, 2015.

[7]. H. K. T. Ng, "Parameter estimation for a modified Weibull distribution, for progressively type-II censored samples," IEEE Transactions on Reliability, vol. 54, no. 3, pp. 374-380, 2005.

[8]. Y. Hak-Keung and T. Siu-Keung, "Parameters estimation for weibull distributed lifetimes under progressive censoring with random removeals,", Journal of Statistical Computation and Simulation, vol. 55, no. 1-2, pp. 57-71, 1996

[9]. S.-J. Wu, "Estimations of the parameters of the Weibull distribution with progressively censored data," Journal of the Japan Statistical Society, vol. 32, no. 2, pp. 155-163, 2002. [10]. H. Ng, P. Chan and N. Balakrishnan, "Estimation of parameters from progressively censored data using EM algorithm," Computational Statistics \& Data Analysis, vol. 39, no. 4, pp. 371-386, 2002.

[11]. M. Nassar, O. Abo-Kasem, D. Sanku and Z. Chunfang, "Analysis of Weibull Distribution Under Adaptive Type-II Progressive Hybrid Censoring Scheme," The Indian Society for Probability and Statistics, vol. 19, no. 1, pp. 25-65, 2018. [12]. S. Ghafouri, A. Habibi Rad and F. Yousefzadeh, "Twosample prediction for progressively Type-II censored Weibull lifetimes," Communications in Statistics Simulation and Computation, vol. 46, no. 2, pp. 1381-1400, 2017.

[13]. T. N. Hon Keung, İ. Kınacı, C. Kuş and c. Ping Sing, "Optimal experimental plan for multi-level stress testing with Weibull regression under progressive Type-II extremal censoring," Communications in Statistics - Simulation and Computation, vol. 46, no. 4, pp. 2611-2637, 2017.

[14]. K. S. Sultan, N. H. Alsadat and D. Kundu, "Bayesian and maximum likelihood estimations of the inverse Weibull parameters under progressive type-II censoring," Journal of Statistical Computation and Simulation, vol. 84, no. 10, pp. 2248-2265, 2014.

[15]. M. M. A. Sobhi and A. A. Soliman, "Estimation for the exponentiated Weibull model with adaptive Type-II progressive censored schemes," Applied Mathematical Modelling, vol. 40, no. 2, pp. 1180-1192, 2016.

[16]. W. Weibull, "A Statistical distribution function of Wide Applicability," ASME Journal of applied Mechanics, pp. 293-297, 1951.

[17]. D. E. Goldberg and J. H. Holland, "Genetic algorithms and machine learning," Machine learning, vol. 3, no. 2, pp. 95-99, 1988.

[18]. R. Luis Miguel and N. V. Sahinidis, "Derivative-free optimization: a review of algorithms and comparison of software implementations," Journal of Global Optimization, vol. 56, no. 3, pp. 1247-1293, 2013.

[19].N. Balakrishnan and R.A.Sandhu, "A Simple Simulation algorithm for Generating Progressive Type-II Censored Samples," The American Statistician, vol. 2, no. 49, pp. 229-230, 1995.

[20]. M. Burkschat, E. Cramer and U. Kamps, "Optimality criteria and optimal schemes in progressive censoring," 
Communications in Statistics-Theory and Methods, vol. 36, no. 7, pp. 1419-1431, 2007.

[21]. J. Lawless, Statistical models and methods for lifetime data, New York: Wiley, 2003.

[22]. C. G. Broyden, "Quasi-Newton methods and their application to function minimisation," Mathematics of Computation, vol. 21, no. 99, pp. 368-381, 1967.
[23]. D. F. Shanno, "Conditioning of quasi-Newton methods for function minimization," Mathematics of computation, vol. 24, no. 111, pp. 647-656, 1970.

[24]. R. Fletcher and M. Powell, "A rapidly convergent descent method for minimization," The computer journal, vol. 6, no. 2, pp. 163-168, 1963. 